\title{
Associated Strengthening Exercises to Undenatured Oral Type II Collagen (UC-II). A Randomized Study in Patients Affected by Knee Osteoarthritis
}

\author{
A. P. Costa1, V. Cunha Teixeira², M. Pereira ${ }^{3}$, P. Mota Ferreira ${ }^{3}$, P. A. Kuplich 4 , M. B. \\ Dohnert $^{5}$, J. F. da Silva Guths ${ }^{6}$, R. Boff Daitx ${ }^{6}$ \\ 1 Department of Physiotherapy, Lutheran University of Brazil, Torres (RS), Brazil \\ 2 Physiotherapist, Torres (RS), Brazil \\ 3 Physiotherapist, Capão da Canoa (RS), Brazil \\ 4 Private Practice, Department of Orthopedics and Traumatology, Santa Luzia Hospital, Capão da Canoa, RS, \\ Brazil \\ ${ }^{5}$ Research, Teaching and Extension Laboratory in Orthopedic Trauma Physiotherapy (LAPEFITO), Department of \\ Physiotherapy, University of Gurupi - UnirG, Gurupi (TO), Brazil \\ 6 Department of Physiotherapy, Lutheran University of Brazil, Torres (RS), Brazil
}

\section{CORRESPONDING AUTHOR:}

Marcelo Baptiista Dohnert

Research, Teaching and Extension

Laboratory in Orthopedic

Trauma Physiotherapy (LAPEFITO)

Department of Physiotherapy

University of Gurupi - UnirG

355 Joaquim Batista de Oliveira Street

Vila Alagoana

77.403-170

Gurupi/TO, Brazil

E-mail: mdohnert@hotmail.com

DOI:

10.32098/mltj.03.2020.18

LEVEL OF EVIDENCE: $1 \mathrm{~B}$

\section{SUMMARY}

Background. Osteoarthritis $(\mathrm{OA})$ is a chronic joint disease characterized by progressive degeneration of articular cartilage. It affects $20-25 \%$ of the population older than 45 years. The objective was to evaluate the effect of combining muscle strengthening exercises with the administration of oral type II undenatured collagen formulation (UC-II) in people with knee OA.

Methods. A double-blind, placebo-controlled, randomized controlled trial with 60 patients with knee OA, randomly divided into the following groups: UC-II and physiotherapy group (CPG), placebo UC-II and physiotherapy group (PCPG), and physiotherapy group (PG). For groups using an oral type II undenatured collagen formulation, UC-II, $40 \mathrm{mg} /$ day UC-II was administered for 90 days. Muscle strengthening exercises were associated with neuromuscular electrical stimulation (NMES), being performed three times a week for 30 days. At 30 and 90 days and six months after the intervention, the following were evaluated: pain, quality of life, functional capacity, muscle strength, and joint mobility.

Results. Pain improved in all intervention groups, with no difference between groups. Quadriceps muscle strength increased in the CPG $(\mathrm{p}<0.005)$ and PG $(\mathrm{p}<0.05)$, the same being observed for active and passive knee flexion mobility $(\mathrm{p}<0.05)$. All groups decreased the TUG test execution time after 30 days $(\mathrm{p}<0.005$ for the CPG and PG, and $p<0.05$ for the PCPG), but only the CPG and PG maintained the scores at 90-day and six-month assessments $(\mathrm{p}<0.005)$. Regarding the 6MWT, only the CPG increased the distance covered in all assessments $(\mathrm{p}<0.005)$. The PG traveled a greater distance than the PCPG at the 90-day assessment $(\mathrm{p}<0.05)$. The WOMAC score decreased significantly in all intervention groups. The Lequesne score decreased in all groups; however, the CPG and PG showed lower values at 30 days $(\mathrm{p}<0.005)$.

Conclusions. Muscle strengthening exercises improved pain, mobility, strength, and function in knee OA patients. The association of UC-II seems to have accentuated the effect of exercise on this clinical improvement, especially in the long term.

KEY WORDS

Knee; osteoarthritis; Circuit-Based Exercise; nutraceuticals; collagen type II; muscle strength. 


\section{BACKGROUND}

Osteoarthritis $(\mathrm{OA})$ is a chronic joint disease characterized by progressive degeneration of articular cartilage accompanied by subchondral bone remodeling and subsequent joint dysfunction (1). Approximately $42 \%$ of women and $31 \%$ of men over 60 are diagnosed with knee OA (2). Knee joint is the most commonly affected lower limb joint $(3,4)$.

Knee OA treatments can be divided into surgical and nonsurgical (5). Results of pain and function improvement are more related to exercise than to pharmacological treatments (5). Thus, therapeutic exercise is considered a first-line treatment for OA (6). Exercise intervention for patients with knee OA should be broad and include lower limb strengthening, improving flexibility to reduce stiffness, increasing stability and balance, reducing body weight, in addition to a psychosocial intervention $(6,7)$.

Type II collagen is the dominant collagen in articular cartilage (8-11). As collagen is the most prevalent component of the solid matrix of articular cartilage, its supplementation has been considered a key treatment option to prevent progressive cartilage damage over time and accelerate the healing process after OA onset (11-14). For this purpose, a new nutraceutical has been studied in individuals with knee OA. It is the undenatured type II collagen (UC-II), taken from chicken sternal cartilage (12).

The present study evaluates the effect of combining muscle strengthening exercises with the administration of an oral type II undenatured collagen formulation (UC-II) in patients with knee OA.

\section{MATERIAL AND METHODS}

A double-blind, placebo-controlled, randomized controlled trial with 60 patients with grade 1-3 knee OA according to the Kellgren Lawrence radiological classification, conducted from April 2015 to May 2019.

\section{Evaluation protocol}

Knee OA was clinically and radiologically diagnosed by an orthopedist of the orthopedics and traumatology service at a regional hospital (table I)

Table I. Elegibility criteria.

\begin{tabular}{ll}
\hline Inclusion Criteria & $\begin{array}{l}\text { Men and women aged } 40 \text { to } 75 \text { years, with unilateral and/or bilateral grade 1-3 knee OA (according to the } \\
\text { Kellgren and Lawrence radiological classification) for more than three months, clinically and radiologically } \\
\text { diagnosed by an orthopedist. }\end{array}$ \\
\hline Ambulatory patients. \\
\hline Lequesne functional score greater than 4.5. \\
\hline Availability during the study period (six months). \\
\hline Not participating in any other physical/drug treatment for the knee during the study period. \\
\hline Having signed the Informed Consent Form. \\
\hline $\begin{array}{l}\text { Patients with OA intensity above grade } 6 \text { according to the Kellgren and Lawrence classification, as confirmed } \\
\text { by an orthopedist. }\end{array}$ \\
\hline Nonambulatory patients. \\
\hline $\begin{array}{l}\text { History of inflammatory arthropathy, septic arthritis, rheumatoid arthritis, inflammatory joint disease, uric } \\
\text { gout, joint fracture, fibromyalgia, collagen genetic disease, neurological disorders, cognitive deficits, history of } \\
\text { asthma, type I or II diabetes, psychiatric disorders, and symptomatic heart disease. }\end{array}$ \\
\hline Clinical condition restricting exercise. \\
\hline Presence of skin injury. \\
\hline Skin disease in the thigh. \\
\hline Urinary incontinence. \\
\hline Pregnancy. \\
\hline Alcoholism. \\
\hline Cancer. \\
\hline Previous history of corticosteroid joint infiltration or knee viscosupplementation in the last three months. \\
\hline History of knee trauma or surgery in the last six months. \\
\hline Having three consecutive unexcused absences from physical rehabilitation. \\
\hline
\end{tabular}


After agreeing to participate in the study, participants were randomized by an independent researcher and referred for a functional physical evaluation performed by a blind assessor previously trained with the evaluation instruments. This professional was not aware of the intervention group to which the participant belonged.

Evaluations were performed prior to randomization, 30 , 90 and six months after the end of the intervention protocol with UC-II. The same evaluator throughout the study always performed all evaluations. The Visual Analog Pain Scale (VAS) was used to assess pain. Knee joint mobility was determined by passive and active flexion and extension using a goniometer (brand $\mathrm{Carci}^{\circledR}$ ). Muscle strength was assessed by manual dynamometry. using a push-pull dynamometer (brand Chattanooga ${ }^{\circledR}$ ). The Timed Up \& Go test (TUG) and the 6-minute walk test (6MWT) were used to assess functional capacity. Quality of life and level of functionality were assessed using the LEQUESNE scale and the WOMAC questionnaire for knee osteoarthritis.

\section{Sample calculation}

Pain was used as the primary outcome of the study for sample calculation. Based on the study of Crowley et al. (9), we estimated the mean initial percentage of pain of the study participants to be $100 \%$. The mean final percentage of pain was $60 \%$ for subjects in the intervention groups after 90 days, and $85 \%$ for subjects in the placebo groups after treatment. Using a study power of $80 \%$, a significance level of $95 \%$, and a sample size ratio of 1:1 for all groups, we reached the estimated number of 13 subjects for each intervention group. Believing that the losses and refusals would be around $50 \%$, we reached the initial number of 20 participants for each of the study groups, totaling 60 participants.

\section{Randomization}

Randomization was performed by an independent researcher, who did not participate in intervention and evaluation protocols.

Patients were randomly divided into a collagen + physiotherapy group (CPG), with 20 participants continuously receiving $\mathrm{UC}-\mathrm{II}^{\circledR}$, and muscle strengthening exercises; a placebo collagen + physiotherapy group (PCPG), with 20 participants continuously receiving placebo UC-II ${ }^{\circledR}$, and muscle strengthening exercises; and a physiotherapy group (PG), with 20 participants receiving muscle strengthening exercises.

\section{ETHICAL ASPECTS}

This research has been approved by the IRB of the authors affiliated institutions. The study meets the ethical standards of the journal. (15)

\section{UC-II intervention protocol}

The randomizing researcher was responsible for providing UC-II or placebo UC-II to the researcher responsible for administering it to the participants. Vials containing the compounds were equally standardized so that the compound could not be identified. Both active and placebo UC-I ${ }^{\circledR}$ were administered for 90 days. Each participant received a vial containing $30 \mathrm{UC}-\mathrm{II}^{\circledR}$ capsules every 30 (thirty) days. Each UC-II ${ }^{\circledR}$ capsule contained $40 \mathrm{mg}$ UC-II ${ }^{\circledR}$ standardized to $10 \mathrm{mg}$ of bioactive undenatured type II collagen, identified by specific registration numbers to differentiate them from placebos. Participants in both groups were instructed to take one UC-II ${ }^{\circledast}$ capsule for breakfast for a daily dose of $40 \mathrm{mg}$ UC-II ${ }^{\circledR}$. Participants in the placebo group received cornstarch capsules. The researcher who administered the nutraceutical intervention, as well as the researcher who administered the physiotherapy and the researcher who performed the evaluations were not aware of which compound was assigned to each participant, only the randomizing researcher had such information.

\section{Physiotherapy intervention protocol}

The physiotherapy protocol was carried out for four weeks, with three interventions per week. The protocol was applied by an independent researcher, who was unaware of the administration of the nutraceutical UC-II ${ }^{\circledR}$.

Prior to protocol implementation, all participants performed a warm-up cycling exercise for five minutes. Afterwards, a closed kinetic chain (CKC) muscle strengthening exercise program was performed through a 45 -degree minisquat, associated with the use of neuromuscular electrostimulation (NMES). For NMES administration, a 10-channel electrical stimulator (Neurodyn, Ibramed ${ }^{\circledR}$ ) was used with a carrier frequency of $2500 \mathrm{~Hz}$, in the synchronous mode. Electrodes were fixed of the vastus medialis, vastus lateralis, rectus femoris (femoral quadriceps), femoral biceps, and semitendinosus (hamstring) muscles.

After electrode fixation, a five-minute warm-up protocol was initially performed using a $40-\mathrm{Hz}$ stimulus frequency, a four-second contraction ("on") time, a four-second rise time, a four-second "off" time, and a 12 -second relaxation time. Afterwards, a 70-Hz stimulus frequency was used for 
another 10 minutes, with the same parameters as above. Finally, a $100-\mathrm{Hz}$ stimulus frequency was used for another 10 minutes for maximum muscle activation, keeping the same parameters.

During the period of muscle stimulation by the electric generator, the patient performed a 30-degree knee flexion minisquat, maintaining this position during the electrical stimulus, returning to full extension at the beginning of the "off" electrostimulation period. During the "off" period, the participant spontaneously performed another active minisquat without the presence of electrical stimulus, returning to the initial position until a new contraction generated by the electrical stimulator.

\section{STATISTICAL ANALYSIS}

The Statistical Package for Social Sciences (SPSS) version 23.0 was used for data analysis. Data were initially expressed as frequency, mean, median, and standard deviation. Afterwards, the normality of data distribution was analyzed using the Shapiro-Wilk test.

For intragroup comparative analysis (CPG, PCPG, and PG) at the various times of the study, parametric data were statistically analyzed by one-way analysis of variance (ANOVA) for repeated measures, followed by the Bonferroni post- hoc test. Moreover, one-way analysis of variance (ANOVA) followed by the Tukey post-hoc test was performed for intergroup analysis, both at preintervention and 30 days, 90 days, and six months after the intervention protocol. For nonparametric variables, the Kruskal Wallis test was used between groups, and the Friedman test was used for analysis within each group. The significance level established for all statistical tests was $\mathrm{p}<0.05$.

\section{RESULTS}

Eighty-four (84) patients were initially selected for the study. Of these, 24 participants were excluded prior to randomization because they did not meet the eligibility criteria. Therefore, the initial sample had a total of 60 participants. All participants completed the 30-day and 90-day assessment. In the follow-up assessment, one CPG participant was not found, totaling a final sample of 59 participants (figure 1).

The groups were homogeneous for anthropometric characteristics, OA grade classification, pain duration, and affected knee (table II).

All intervention groups significantly reduced pain after 30 days of intervention, maintaining this improvement until the follow-up assessment. There were no differences between groups at any time of the study. The CPG reduced pain

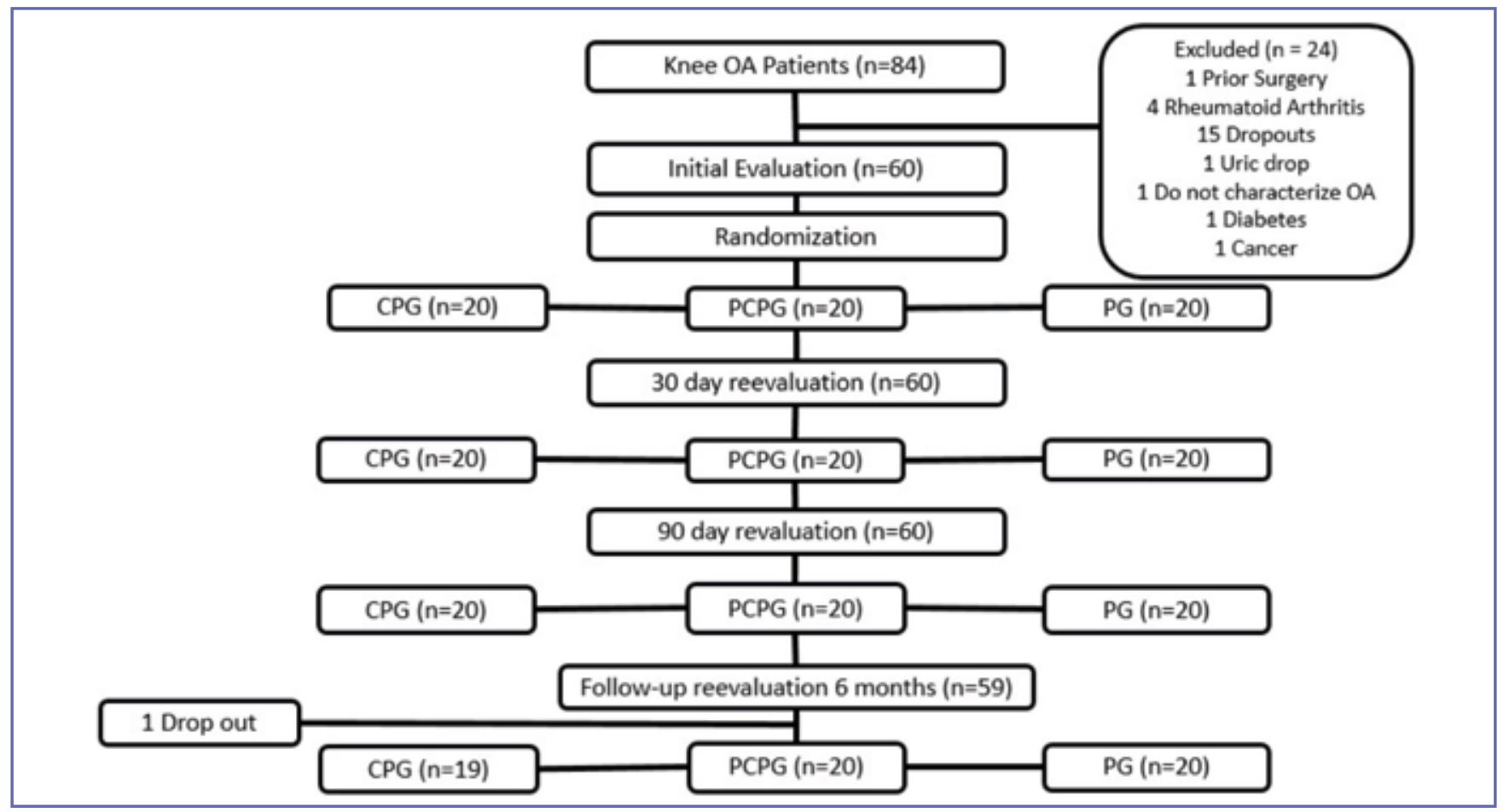

Figure 1. Study flowchart. 
Table II. Characterization of the initial study sample $(n=60)$.

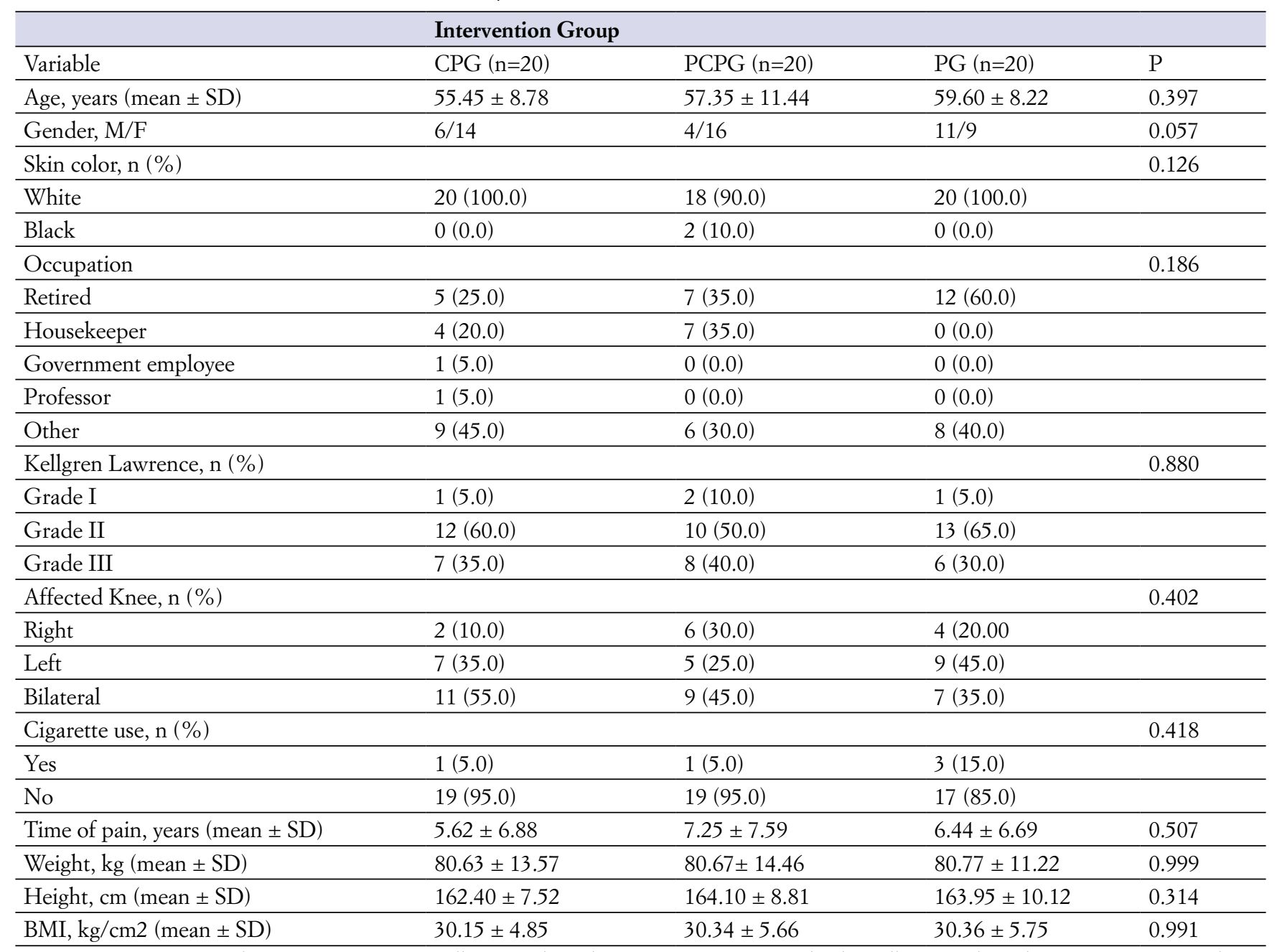

\#: One-way ANOVA; \$: Chi-square test; CPG = collagen + physiotherapy group; PCPG = placebo collagen + physiotherapy group; PG = physiotherapy group.

from $6.42 \pm 1.68$ points at baseline to $1.42 \pm 1.77$ at 30 days $(\mathrm{p}=0.0001), 2.74 \pm 2.56$ at 90 days $(\mathrm{p}=0.0001$ compared to baseline), and $3.26 \pm 2.88$ after six months ( $\mathrm{p}=0.0001$ compared to baseline). The PCPG showed an initial pain level of $5.60 \pm 2.11$ points. At the 30-day assessment, pain was reduced to $2.65 \pm 2.62(\mathrm{p}=0.0001)$. At the 90 -day assessment, the pain level was $2.75 \pm 2.53(\mathrm{p}=0.0001$ compared to the initial assessment). After six months, the pain level was $3.05 \pm 2.82$ ( $\mathrm{p}=0.01$ compared to the initial assessment). Finally, the PG showed an initial pain level of $6.10 \pm 1.91$ points, which decreased to $1.80 \pm 2.28$ at the 30 -day assessment $(\mathrm{p}=0.0001)$. At the 90-day assessment, the pain level was $2.35 \pm 2.46(\mathrm{p}=0.0001$ compared to the initial assessment), remaining at $2.30 \pm 2.00$ after six months $(\mathrm{p}=0.0001$ compared to the initial assessment) (figure 2).
The PG demonstrated a significantly higher initial right quadriceps strength compared to the CPG. The CPG, however, significantly increased muscle strength at 30 days. The other groups did not improve muscle strength. At the 90-day and six-month assessments, the PG showed significantly greater muscle strength than the CPG and PCPG. Regarding left quadriceps muscle strength, only the CPG and PG increased muscle strength at 30 days, maintaining an improved strength at the 90-day and six-month assessments. The PG showed a significantly higher level of muscle strength than the CPG at the initial evaluation. At 30 days, the PG had a significantly higher level of left quadriceps strength than the PCPG $(\mathrm{p}<0.05)$. At the 90-day and six-month assessments, the PG showed higher muscle strength than the other groups (table III). 


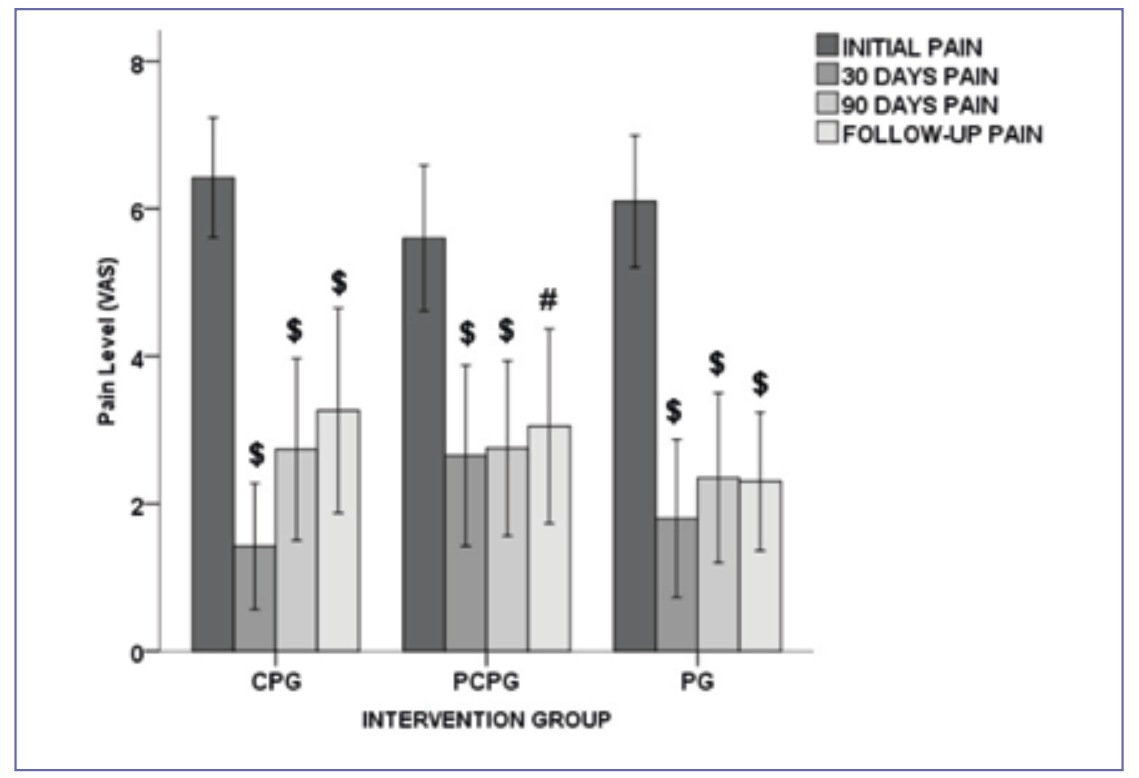

Figure 2. Pain assessment in the study groups. $\# p<0.05$ compared to the initial assessment of the same group. ANOVA for repeated measures.

$\$ p<0.005$ compared to the initial assessment of the same group. ANOVA for repeated measures.

Table III. Results of quadriceps and hamstring muscle strength (MVIC) in the initial study groups, 30 days, 90 days, and six months after UC-II administration $(n=60)$.

\begin{tabular}{|c|c|c|c|c|}
\hline & Intervention $\mathrm{G}$ & & & \\
\hline Variable & CPG $(n=20)$ & PCPG $(n=20)$ & $P G(n=20)$ & $\mathrm{P}$ \\
\hline \multicolumn{5}{|c|}{ Right quadriceps, $\mathrm{kg} \pm \mathrm{sd}$} \\
\hline Initial & $17.71 \pm 7.05$ & $18.15 \pm 7.54$ & $27.60 \pm 12.39$ & $0.02^{\mathrm{b}}$ \\
\hline 30 days & $24.45 \pm 10.59^{\$}$ & $22.50 \pm 9.39$ & $29.20 \pm 12.17$ & 0.14 \\
\hline 90 days & $23.10 \pm 10.24^{\#}$ & $21.90 \pm 9.19$ & $30.55 \pm 11.82$ & $0.02^{\mathrm{a}}$ \\
\hline Follow-up & $23.32 \pm 11.29$ & $22.50 \pm 10.05$ & $31.05 \pm 11.24$ & $0.03^{\mathrm{a}}$ \\
\hline \multicolumn{5}{|c|}{ Left quadriceps, $\mathrm{kg} \pm \mathrm{sd}$} \\
\hline Initial & $16.33 \pm 6.87$ & $19.45 \pm 8.35$ & $25.20 \pm 11.17$ & $0.01^{\mathrm{b}}$ \\
\hline 30 days & $24.15 \pm 10.30^{\$}$ & $21.95 \pm 9.86$ & $30.10 \pm 10.98^{\#}$ & $0.04^{c}$ \\
\hline 90 days & $23.15 \pm 10.95^{\$}$ & $22.10 \pm 9.42$ & $30.15 \pm 10.62^{\#}$ & $0.03^{\mathrm{a}}$ \\
\hline Follow-up & $22.95 \pm 9.26^{\$}$ & $21.95 \pm 8.70$ & $30.55 \pm 10.92^{\#}$ & $0.01^{\mathrm{a}}$ \\
\hline \multicolumn{5}{|c|}{ Right hamstrings, $\mathrm{kg} \pm \mathrm{sd}$} \\
\hline Initial & $12.41 \pm 4.60$ & $12.10 \pm 6.18$ & $15.10 \pm 6.95$ & 0.30 \\
\hline 30 days & $14.20 \pm 5.30$ & $13.40 \pm 5.81$ & $16.15 \pm 6.66$ & 0.33 \\
\hline 90 days & $13.55 \pm 5.61$ & $13.50 \pm 5.21$ & $16.50 \pm 5.24$ & 0.14 \\
\hline Follow-up & $14.79 \pm 6.49$ & $14.10 \pm 8.47$ & $16.20 \pm 5.50$ & 0.62 \\
\hline \multicolumn{5}{|c|}{ Left hamstrings, $\mathrm{kg} \pm \mathrm{sd}$} \\
\hline Initial & $11.15 \pm 4.85$ & $12.35 \pm 6.29$ & $15.00 \pm 6.23$ & 0.11 \\
\hline 30 days & $13.85 \pm 5.24$ & $13.80 \pm 6.11$ & $17.50 \pm 4.82$ & $0.05^{\mathrm{a}}$ \\
\hline 90 days & $16.65 \pm 15.89$ & $13.85 \pm 5.21$ & $16.50 \pm 5.24$ & 0.55 \\
\hline Follow-up & $13.84 \pm 6.21$ & $13.75 \pm 5.73$ & $16.05 \pm 5.11$ & 0.59 \\
\hline
\end{tabular}

$\mathrm{CPG}=$ collagen + physiotherapy group $\mathrm{PCPG}=$ placebo collagen + physiotherapy group; $\mathrm{PG}=$ physiotherapy group.

\# $\mathrm{p}<0.05$ compared to the initial assessment of the same group. ANOVA for repeated measures.

$\$ \mathrm{p}<0.005$ compared to the initial assessment of the same group. ANOVA for repeated measures.

a $\mathrm{p}<0.05$ between the PG and the others. One-way ANOVA.

$\mathrm{b} p<0.05$ between the PG and the CPG. One-way ANOVA.

c $\mathrm{p}<0.05$ between the PG and the PCPG. One-way ANOVA. 
Right hamstring muscle strength did not differ significantly between groups and in each group throughout the intervention protocol. For left hamstrings, the PG showed a higher muscle strength than the other groups at the 30-day assessment (table III).

There were no differences in active and passive bilateral knee ROM between the study groups. Active flexion range of motion of the right knee increased in the CPG and PG. For the left knee, ROM increased only in the CPG. For passive flexion ROM of the right knee, only the PG increased mobility after 90 days. Passive flexion ROM of the left knee did not change throughout the intervention protocol (table IV).

Active extension of both knees did not differ between the groups and throughout the intervention protocol in the study groups (table IV).

All intervention groups reduced the TUG execution time at the 30-day assessment, with no differences between groups. However, at the 90-day and six-month assessments, the PCPG did not maintain this improvement. The CPG showed an initial score of $11.53 \pm 3.51$ seconds, which decreased to $8.91 \pm 2.87$ at 30 days $(\mathrm{p}=0.001)$, remaining at $8.99 \pm 2.93$ at 90 days $(\mathrm{p}=0.0001)$, and $9.24 \pm 3.91$ after six months ( $\mathrm{p}=0.032$ ). The PG decreased the TUG test execution time from $11.30 \pm 3.32$ seconds at baseline to $8.39 \pm$ 2.23 at the 30 -day assessment $(\mathrm{p}=0.0001)$, remaining at 8.52 \pm 2.47 at the 90 -day assessment $(\mathrm{p}=0.001)$, and $9.08 \pm 3.31$ at the six-month assessment $(\mathrm{p}=0.001)$. For the PCPG, the execution time decreased from $11.56 \pm 4.26$ seconds at baseline to $8.85 \pm 2.53$ seconds at the 30 -day assessment $(\mathrm{p}=0.007)$. However, the execution time increased to $9.32 \pm$ 3.51 at 90 days, and to $9.93 \pm 3.14$ at six months (figure 3 ). The PG significantly increased the distance covered in the 6MWT at 90 days compared to the PCPG. Only the CPG and $P G$ increased the distance covered in the test from the 30-day assessment. The CPG initially covered $301.38 \pm$ 92.21 meters. After 30 days, this distance increased to 356.60 $\pm 98.92(\mathrm{p}=0.0001)$. At the 90-day assessment, the distance covered was $346.67 \pm 99.37$ ( $\mathrm{p}=0.001$ compared to the initial assessment). Six months after the intervention, the distance covered was $372.10 \pm 145.04(\mathrm{p}=0.0001)$. The PG covered an initial distance of $333.55 \pm 92.98$ meters. At the 30-day assessment, the distance covered by this group was $394.55 \pm$ 86.11 ( $\mathrm{p}=0.0001$ ). This distance decreased to $390.65 \pm 87.79$ at the 90 -day assessment $(\mathrm{p}=0.0001)$ compared to the initial assessment), and to $349.00 \pm 141.75$ at six months ( $\mathrm{p}=0.001$ ) compared to the initial assessment). The PCPG showed no significant change in the distance covered on the TUG at different times of assessment ( $p>0,05)$ (figure 4)

Functionality and quality of life, as assessed by the Lequesne Algofunctional Questionnaire, increased for the CPG and
PG compared to the PCPG at the postintervention assessment (30 days). All intervention groups improved functionality scores at the 30-day and 90-day assessments. Only the PCPG did not maintain this improvement at the six-month assessment. The CPG presented an initial Lequesne score of $11.18 \pm 3.30$. At the 30-day assessment, the score decreased to $5.18 \pm 4.04$ points $(\mathrm{p}=0.001)$. At the 90 -day assessment, the score was $5.85 \pm 5.10$ points $(\mathrm{p}=0.0001$ compared to the initial assessment). After six months, the score was 7.08 \pm 5.97 ( $\mathrm{p}=0.013$ compared to the initial assessment). On the other hand, the PG showed an initial Lequesne score of $9.33 \pm 2.49$ points. After 30 days, the score decreased to $4.00 \pm 3.25(\mathrm{p}=0.0001)$. The score was $4.68 \pm 4.06$ points $(\mathrm{p}=0.001)$ at the 90 -day assessment, and $5.70 \pm 3.64$ $(\mathrm{p}=0.001)$ after six months. The PCPG had an initial score of $11.20 \pm 3.45$ points, which decreased to $7.25 \pm 4.66$ after 30 days $(\mathrm{p}=0.0001)$, remaining at $7.73 \pm 5.40$ after 90 days $(\mathrm{p}=0.003)$, and $8.75 \pm 6.30$ six months after the intervention $(\mathrm{p}=0.15)$ (figure 5).

All intervention groups reduced the WOMAC Questionnaire scores, with no differences between them. The CPG had an initial score of $45.25 \pm 17.88$ points. The score decreased to $16.75 \pm 15.91$ ( $\mathrm{p}=0.0001)$ at the 30 -day assessment, remaining at $22.35 \pm 22.17$ at the 90 -day assessment ( $\mathrm{p}=0.0001$ compared to the preintervention assessment). Six months after the intervention, the score was $24.11 \pm$ 24.50 ( $\mathrm{p}=0.0001$ compared to the preintervention assessment). The PCPG showed an initial score of $43.00 \pm 20.21$ points, which decreased to $22.90 \pm 19.99$ points at the 30-day assessment $(\mathrm{p}=0.0001)$, remaining at $23.60 \pm 23.75$ at the 90-day assessment $(\mathrm{p}=0.0001)$, and $27.05 \pm 24.76$ at the follow-up assessment ( $\mathrm{p}=0.019$ compared to the initial assessment). The PG showed an initial WOMAC score of $34.65 \pm 11.93$ points. At the 30 -day assessment, the score decreased to $11.35 \pm 11.45(\mathrm{p}=0.0001)$. At the 90-day assessment, the score was $13.35 \pm 12.11(\mathrm{p}=0.0001)$. Six months after the intervention, the score was $12.60 \pm 11.37$ $(\mathrm{p}=0.0001)$ (figure 6).

\section{DISCUSSION}

This study investigated the effect of using the nutraceutical UC-II ${ }^{\circledR}$ combined with a physiotherapy program on symptomatology, joint mobility, muscle strength, and knee joint function in patients with knee OA.

We verified a pain reduction both in the group that administered only UC-II and in the group that performed only muscle strengthening. The association of UC-II with exercises did not demonstrate greater efficacy over pain than each of them administered in isolation. Exercise is strongly recommended for nonsurgical treatment of OA (16). In a 
Table IV. Results of active and passive knee joint range of motion (ROM) in the initial study groups, 30 days, 90 days, and six months after UC-II administration $(n=40)$.

\begin{tabular}{|c|c|c|c|c|}
\hline \multirow[b]{2}{*}{ Variable } & \multicolumn{4}{|c|}{ Intervention Group } \\
\hline & CPG (n=20) & PCPG $(n=20)$ & PG $(n=20)$ & $\mathbf{P}$ \\
\hline \multicolumn{5}{|c|}{ Right active flexion, degrees (mean \pm SD) } \\
\hline Initial & $117.10 \pm 13.71$ & $118.80 \pm 17.06$ & $120.80 \pm 13.52$ & 0.73 \\
\hline 30 days & $126.55 \pm 9.15 \#$ & $125.75 \pm 15.44$ & $123.10 \pm 30.84$ & 0.86 \\
\hline 90 days & $124.85 \pm 13.29$ & $125.25 \pm 13.74$ & $129.05 \pm 11.21 \$$ & 0.53 \\
\hline Follow-up & $124.00 \pm 14.13$ & $122.00 \pm 16.88$ & $128.85 \pm 10.58 \$$ & 0.29 \\
\hline \multicolumn{5}{|c|}{ Left active flexion, degrees $($ mean \pm SD) } \\
\hline Initial & $114.85 \pm 14.42$ & $120.30 \pm 14.68$ & $120.55 \pm 22.15$ & 0.51 \\
\hline 30 days & $124.20 \pm 10.32 \#$ & $124.80 \pm 10.53$ & $130.50 \pm 13.73 \$$ & 0.18 \\
\hline 90 days & $124.65 \pm 12.21 \#$ & $124.95 \pm 11.70$ & $131.20 \pm 14.23$ & 0.20 \\
\hline Follow-up & $124.42 \pm 12.64 \#$ & $122.50 \pm 14.20$ & $130.35 \pm 13.79$ & 0.09 \\
\hline \multicolumn{5}{|c|}{ Right passive flexion, degrees (mean $\pm \mathrm{SD}$ ) } \\
\hline Initial & $127.75 \pm 12.38$ & $125.60 \pm 16.30$ & $128.85 \pm 19.77$ & 0.73 \\
\hline 30 days & $133.60 \pm 9.49$ & $132.70 \pm 10.78$ & $129.55 \pm 32.66$ & 0.81 \\
\hline 90 days & $132.00 \pm 8.94$ & $133.10 \pm 11.77$ & $136.65 \pm 11.78 \$$ & 0.37 \\
\hline Follow-up & $130.79 \pm 11.33$ & $130.85 \pm 15.34$ & $135.90 \pm 12.26 \$$ & 0.38 \\
\hline \multicolumn{5}{|c|}{ Left passive flexion, degrees (mean \pm SD) } \\
\hline Initial & $124.05 \pm 13.87$ & $125.25 \pm 16.30$ & $128.85 \pm 16.66$ & 0.65 \\
\hline 30 days & $132.30 \pm 10.78$ & $131.15 \pm 11.28$ & $137.10 \pm 11.92$ & 0.22 \\
\hline 90 days & $130.90 \pm 12.37$ & $131.75 \pm 11.77$ & $136.85 \pm 12.47$ & 0.24 \\
\hline Follow-up & $130.53 \pm 12.52$ & $130.05 \pm 13.49$ & $136.80 \pm 11.81$ & 0.12 \\
\hline \multicolumn{5}{|c|}{ Right active extension, degrees (mean \pm SD) } \\
\hline Initial & $-1.60 \pm 3.93$ & $-1.15 \pm 2.64$ & $-3.65 \pm 8.83$ & 0.35 \\
\hline 30 days & $-1.05 \pm 3.24$ & $-0.90 \pm 3.58$ & $-0.70 \pm 3.13$ & 0.95 \\
\hline 90 days & $-0.60 \pm 2.26$ & $-0.10 \pm 0.44$ & $-0.75 \pm 3.35$ & 0.66 \\
\hline Follow-up & $-0.53 \pm 2.29$ & $-0.90 \pm 3.58$ & $-0.85 \pm 2.85$ & 0.91 \\
\hline \multicolumn{5}{|c|}{ Left active extension, degrees (mean \pm SD) } \\
\hline Initial & $-3.90 \pm 6.01$ & $-2.95 \pm 6.53$ & $-2.45 \pm 6.24$ & 0.76 \\
\hline 30 days & $-1.00 \pm 3.08$ & $-1.15 \pm 4.12$ & $-0.60 \pm 2.68$ & 0.87 \\
\hline 90 days & $-1.20 \pm 3.14$ & $-0.15 \pm 0.68$ & $-0.75 \pm 3.35$ & 0.39 \\
\hline Follow-up & $-1.89 \pm 4.58$ & $-1.05 \pm 4.05$ & $-0.60 \pm 2.68$ & 0.38 \\
\hline \multicolumn{5}{|c|}{ Right passive extension, degrees $\pm \mathrm{SD}$ ) } \\
\hline Initial & $-1.00 \pm 2.61$ & $-0.40 \pm 1.19$ & $-2.50 \pm 5.74$ & 0.19 \\
\hline 30 days & $-1.05 \pm 3.24$ & $0.40 \pm 1.79$ & $-0.60 \pm 2.68$ & 0.21 \\
\hline 90 days & $0.00 \pm 0.00$ & $0.00 \pm 0.00$ & $-0.50 \pm 2.24$ & 0.37 \\
\hline Follow-up & $-0.53 \pm 2.29$ & $-0.20 \pm 0.89$ & $-0.60 \pm 2.68$ & 0.81 \\
\hline \multicolumn{5}{|c|}{ Left passive extension, degrees \pm SD) } \\
\hline Initial & $-1.95 \pm 3.73$ & $-2.05 \pm 5.42$ & $-1.65 \pm 5.10$ & 0.96 \\
\hline 30 days & $-0.90 \pm 2.79$ & $0.50 \pm 2.24$ & $-0.60 \pm 2.68$ & 0.20 \\
\hline 90 days & $-0.40 \pm 1.79$ & $0.00 \pm 0.00$ & $-0.50 \pm 2.24$ & 0.60 \\
\hline Follow-up & $-1.53 \pm 3.66$ & $-0.40 \pm 1.79$ & $-0.60 \pm 2.68$ & 0.41 \\
\hline
\end{tabular}

$\mathrm{CPG}=$ collagen + physiotherapy group PCPG = placebo collagen + physiotherapy group; $\mathrm{PG}=$ physiotherapy group.

\# $\mathrm{p}<0.05$ compared to the initial assessment of the same group.

$\$ \mathrm{p}<0.005$ compared to the initial assessment of the same group. ANOVA for repeated measures. 


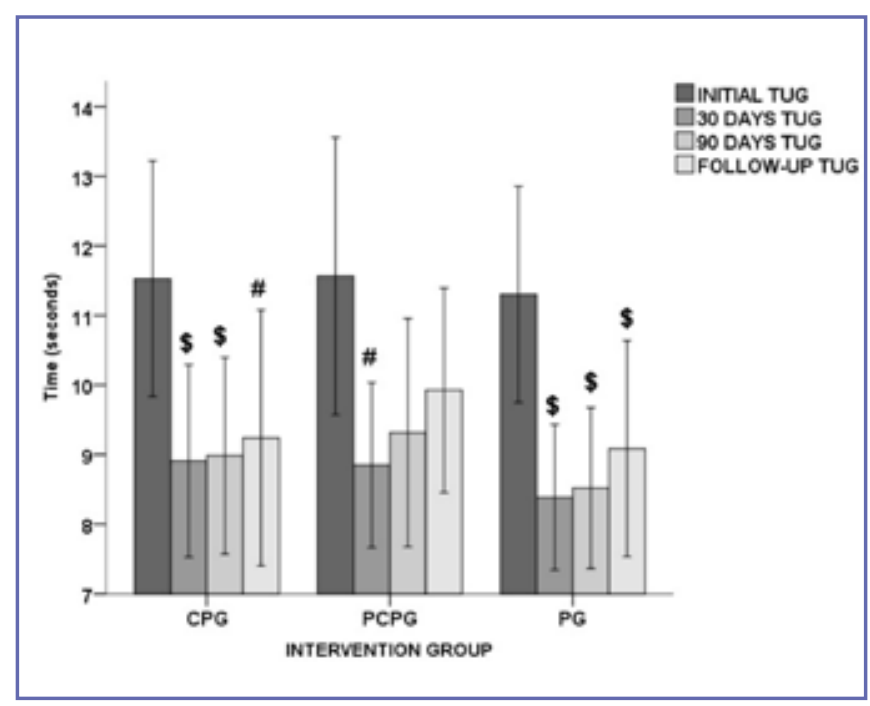

Figure 3. TUG in study groups.

\# $p<0.05$ compared to the initial assessment of the same group. ANOVA for repeated measures.

$\$ p<0.005$ compared to the initial assessment of the same group. ANOVA for repeated measures.

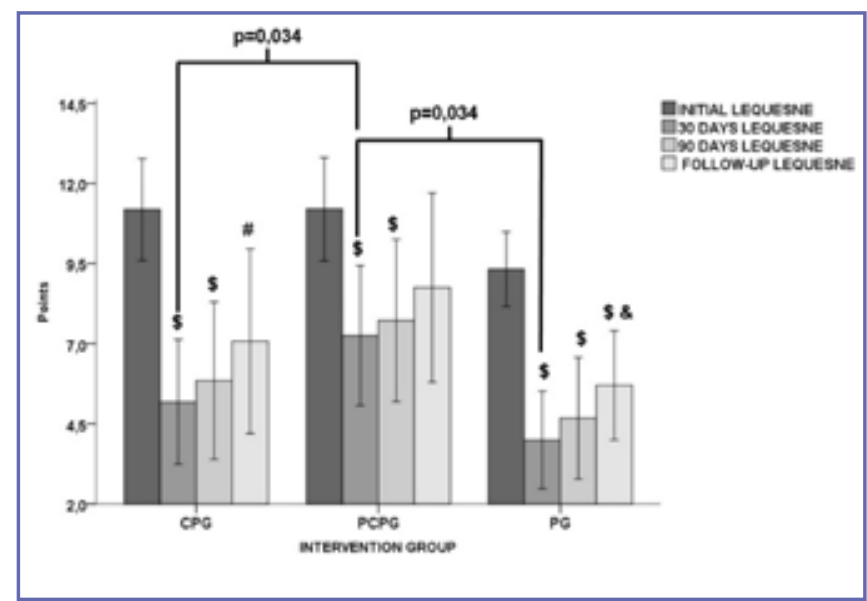

Figure 5. Lequesne score in study groups. One-way ANOVA. $\$ \mathrm{P}<0.005 ; \# \mathrm{p}<0.05$ compared to the initial assessment of the same group.

$\& p<0.005$ compared to the 30 -day assessment of the same group. ANOVA for repeated measures.

study of participants with OA pain exacerbations, strengthening and coordination exercises performed three times a week for 12 weeks reduced pain in $63 \%$ of sessions (17). Pain is the main problem in knee OA because it precedes other negative aspects of the subject's life, such as physical

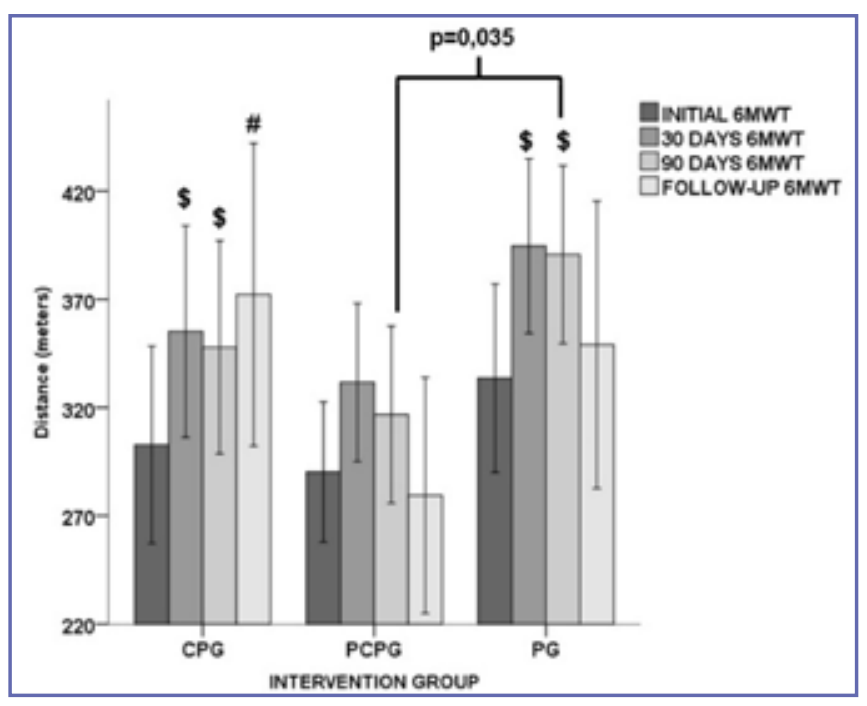

Figure 4. Distance in the 6MWT in study groups. One-way ANOVA.

$\$ \mathrm{P}<0.005$ compared to the initial assessment of the same group. \# $p<0.05$ compared to the initial assessment of the same group. ANOVA for repeated measures.

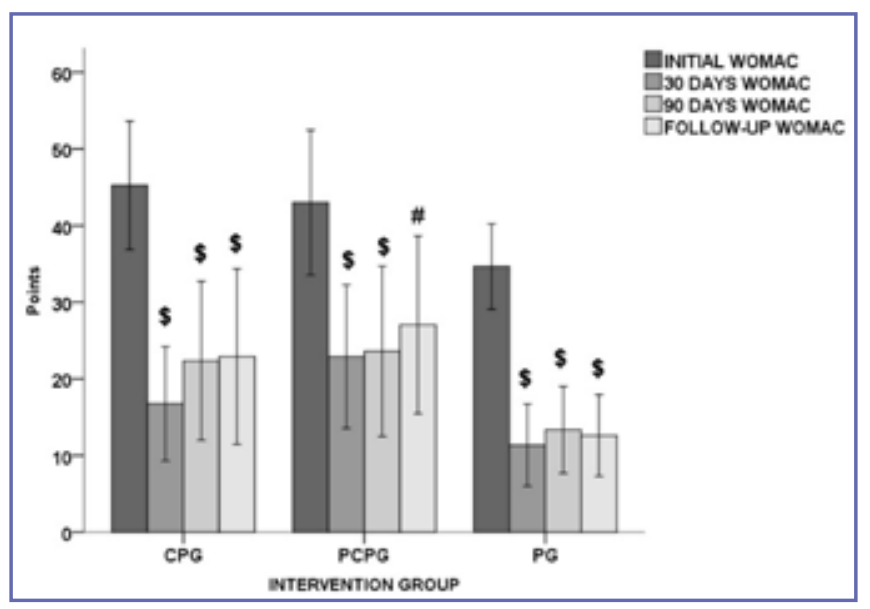

Figure 6. Total WOMAC score in study groups.

\# $p<0.05$ compared to the initial assessment of the same group. ANOVA for repeated measures.

$\$ p<0.001$ compared to the initial assessment of the same group. ANOVA for repeated measures.

inactivity, which contributes to decreased muscle strength and gait changes (18). Notwithstanding, it is important to understand that there is an inflammatory signaling pathway that leads to pain and loss of muscle strength. Inflammatory cytokines such as IL- $1 \beta$ and TNF- $\alpha$ lead to the destruction 
of the extracellular matrix (ECM) of the cartilage locally in the OA joint (19). In addition, IL- $1 \beta$ and TNF- $\alpha$ contribute to NF-kB activation in synovial cells and chondrocytes, both commonly implicated in OA pathogenesis (19). Another important factor that can be attributed to pain improvement after exercise is the facilitation of endorphin release, which makes the patient more tolerant to it $(20,21)$.

The effectiveness of therapeutic ground exercise for people with knee and hip OA has been investigated in terms of pain reduction and disability (22). Nevertheless, few studies have investigated the specificity of the dose, frequency, and intensity of these exercises for knee OA and their effects on pain reduction (22). This omission of essential information on exercise interventions for knee OA is quite problematic and may result in significant clinical uncertainty, variability, and limited implementation, although global recommendations converge to focus on exercises in the therapeutic approach to OA (7). There is a need to specify recommendations and replicate the original studies with close attention to intervention protocols (21). Right quadriceps muscle strength increased by $38 \%$ in the CPG after exercise. Left quadriceps strength increased by 48 and $20 \%$, respectively, in the PCG and PG. A 30\% increase in knee extensor strength is required to improve pain outcomes (16). In this study, both the group that administered UC-II and the group that performed exercises increased quadriceps strength after interventions. However, the group that administered the exercises showed a quadriceps strength significantly greater than the other intervention groups. Pain reduction after strengthening exercises can be attributed to reversal of quadriceps weakness, which is one of the main conditions present in patients with knee OA (22) Strength training can increase muscle strength and reduce pain at the same time (20)

Stratford, Kennedy and Woodhouse (23) found that the 6MWT and TUG demonstrated two factors consistent with health concepts linked to pain and function of patients with knee and hip OA. Ateef, Kulandaivelan and Tahseen (24) approached 80 people with knee $\mathrm{OA}$ and observed that women walked 260.20 meters, while men walked 327.38 meters. Knee pain affects the ability to walk or perform weight-bearing exercises, thus reducing exercise adherence, compromising the goal of improving body composition $(25,26)$. In our study, we found an improvement in the TUG test in the CPG and PG groups in all evaluations, which demonstrates that exercises were largely responsible for the functional improvement of patients with knee OA. Regarding 6MWT, the same groups increased the distance covered. In the present study, there was no significant potential effect of UC-II when associated with muscle strengthening exercise. However, the group that combined the two interventions showed more robust results, especially six months after the intervention protocol. UC-II has been shown to be able to "turn off" the specifically targeted immune response to articular cartilage (type II) collagen, without presenting adverse effects $(27,28)$.

Osteoarthritis is histologically characterized by changes in chondrocyte cell structure and loss of proteoglycans (29). Other histological features of OA include synovial membrane hyperplasia and thickening, infiltration of inflammatory cells, and fibrosis (29). In advanced stages of the disease, the cartilage shows signs of complete rupture, so that hyaline cartilage is replaced by a scar-like fibrocartilaginous tissue with fibroblast-like cells (29). Nutraceuticals play an important role in the anabolic and catabolic balance of articular cartilage. The association of these with the therapies already commonly postulated increases the therapeutic options in knee OA. Few researches already carried out demonstrated that the treatment of patients with OA with UC-II increases the mobility and functionality of the joints and reduces pain (29). Fusini et al. conducted a comprehensive review to analyze the use of nutrients in the treatment of tendinopathies (30). However, the authors have failed to establish a recommendation for the use of these to address tendinopathies (30). Type 1 hydrolyzed collagen can increase the amount of dermatansulfate and decrease levels of hyaluronic acid, especially at high doses (30).

This is the first study to specifically analyze the effect of joint administration of UC-II and muscle strengthening exercises. Thus, patients, physicians, and physiotherapists should have an objective realistic outcome, with a combination of these therapeutic options being the most preferable scenario, particularly considering the available evidence for nonpharmacological management.

\section{LIMITATIONS}

We recognize some limitations of the study. This study did not have a control group for an adequate understanding of disease progression. All participants who entered the clinical trial volunteered to participate, indicating motivation to participate in one of the intervention groups. Therefore, the results may not be generalizable to other adults with knee OA. A standard exercise program, specific for strengthening the quadriceps, was used. We believe that other exercise techniques, in conjunction with UC-II, should be performed to find a better therapeutic option for patients with knee OA.

\section{CONCLUSIONS}

The results of this study suggest that a 30-day NMES-associated muscle strengthening program was effective in improv- 
ing pain, strength, and function in people with knee OA. Combining the nutraceutical UC-II with muscle strengthening exercises seems to have potentiated the effect of muscle strengthening, especially in the long term.

\section{ACKNOWLEDGEMENTS}

To the Infinity Pharma ${ }^{\circledR}$ Laboratory in Campinas/SP, Brazil, for the partial funding of this study.

\section{REFERENCES}

1. Zhang X, Yang Y, Li X, Zhang H, Gang Y, Bai L. Alterations of autophagy in knee cartilage by treatment with treadmill exercise in a rat osteoarthritis model. Int $\mathrm{J} \mathrm{Mol} \mathrm{Med}$ 2019;43(1):336-44.

2. Rabe KG, Matsuse H, Jackson A, Segal NA. Evaluation of the Combined Application of Neuromuscular Electrical Stimulation and Volitional Contractions on Thigh Muscle Strength, Knee Pain, and Physical Performance in Women at Risk for Knee Osteoarthritis: A Randomized Controlled Trial PM R. 2018;10(12):1301-10.

3. Hall M, Castelein B, Wittoek R, Calders P, Ginckel A Van. Diet-induced weight loss alone or combined with exercise in overweight or obese people with knee osteoarthritis: a systematic review and meta-analysis. Semin Arthritis Rheum 2019; 48(5): $765-777$.

4. Van Ginckel A, Hall M, Dobson F, Calders P. Effects of longterm exercise therapy on knee joint structure in people with knee osteoarthritis: A systematic review and meta-analysis. Semin Arthritis Rheum 2019; 48(6): 941-949.

5. Kan HS, Chan PK, Chiu KY, Yan CH, Yeung SS, Ng YL, Shiu KW, Ho T. Non-surgical treatment of knee osteoarthritis. Hong Kong Med J. 2019; 25(2): 127-133

6. McKay J, Frantzen K, Vercruyssen N, Hafsi K, Opitz T, Davis A, Murrell W. Rehabilitation following regenerative medicine treatment for knee osteoarthritis-current concept review. J Clinl Orthop Trauma 2019; 10(1):59-66.

7. Bartholdy C, Nielsen SM, Warming S, Hunter DJ, Christensen R, Henriksen M. Poor replicability of recommended exercise interventions for knee osteoarthritis: a descriptive analysis of evidence informing current guidelines and recommendations. Osteoarthritis Cartilage 2019;27(1):3-22.

8. Quicke JG, Foster NE, Croft PR, Ogollah RO, Holden MA. Change in physical activity level and clinical outcomes in older adults with knee pain: A secondary analysis from a randomised controlled trial. BMC Musculoskelet Disord 2018; 19(1):59.

9. Crowley DC, Lau FC, Sharma P, Evans M, Guthrie N, Bagchi M, Bagchi D, Dey DK, Raychaudhuri SP. Safety and efficacy of undenatured type II collagen in the treatment of osteoarthritis of the knee: a clinical trial. Int J Med Sci 2009; 6(6):312-21.

10. Lugo JP, Saiyed ZM, Lane NE. Efficacy and tolerability of an undenatured type II collagen supplement in modulating knee osteoarthritis symptoms: a multicenter randomized, doubleblind, placebo-controlled study. BMC Nutrition Journal 2016; 11(1):1-15.
To the Sea Life ${ }^{\circledR}$ handling pharmacy in Torres/RS, Brazil, represented by the pharmacist-in-charge Josele Milanez Paz. To the Group of Studies and Research in Sports Rehabilitation and Orthopedic Trauma (GEPRETO) of the Lutheran University of Brazil, Torres/RS Campus, Brazil.

\section{CONFLICT OF INTERESTS}

The authors declare that they have no conflict of interests.

11. Bagi CM, Berryman ER, Teo S, Lane NE. Oral administration of undenatured native chicken type II collagen (UC-II) diminished deterioration of articular cartilage in a rat model of osteoarthritis (OA). Osteoarthritis Cartilage. 2017; 25(12):2080-2090.

12. Lewek MD, Rudolph KS, Snyder-Mackler L. Quadriceps femoris muscle weakness and activation failure in patients with symptomatic knee osteoarthritis. J Orthop Res 2004; 22(1):110-5.

13. Taş S, Güneri S, Baki A, Yildirim T, Kaymak B, Erden Z. Effects of severity of osteoarthritis on the temporospatial gait parameters in patients with knee osteoarthritis. Acta Orthop Traumatol Turc 2014; 48(6):635-41.

14. Lugo JP, Saiyed ZM, Lau FC, Molina JPL, Pakdaman MN, Shamie AN, Udani JK. Undenatured type II collagen (UC-II®) for joint support: A randomized, double-blind, placebo-controlled study in healthy volunteers. J Int Soc Sports Nutr 2013;10(1):1.

15. Padulo J, Oliva F., Frizziero A, Maffulli N. Basic principles and recommendations in clinical and field Science Research: 2018 update. MLTJ 2018; 8(3): 305 - 307.

16. Wellsandt E, Golightly Y. Exercise in the management of knee and hip osteoarthritis. Curr Opin Rheumatol 2018; 30(2):151-9.

17. Bartholdy C, Klokker L, Bandak E, Bliddal H, Henriksen M. A Standardized "Rescue" Exercise Program for Symptomatic Flare-up of Knee Osteoarthritis: Description and Safety Considerations. J Orthop Sport Phys Ther 2016; 46(11):942-946.

18. Braghin R de MB, Libardi EC, Junqueira C, Nogueira - Barbosa $\mathrm{MH}$, de Abreu DCC. Exercise on balance and function for knee osteoarthritis: A randomized controlled trial. J Bodyw Mov Ther 2018; 22(1):76-82.

19. Krishnasamy P, Hall M, Robbins SR. The role of skeletal muscle in the pathophysiology and management of knee osteoarthritis. Rheumatol (Oxford). 2018; 57(Suppl_4): iv22-iv33.

20. Bokaeian HR, Bakhtiary AH, Mirmohammadkhani M, Moghimi J. Quadriceps strengthening exercises may not change pain and function in knee osteoarthritis. J Bodyw Mov Ther 2018; 22(2): 528-533.

21. Marone PA, Lau FC, Gupta RC, Bagchi M, Bagchi D. Safety and toxicological evaluation of undenatured type II collagen. Toxicol Mech Methods 2010; 20(4):175-89.

22. Imoto AM, Pardo JP, Brosseau L, Taki J, Desjardins B, Thevenot O, Franco E, Peccin S. Evidence synthesis of types 
and intensity of therapeutic land-based exercises to reduce pain in individuals with knee osteoarthritis. Rheumatol Int 2019; 39(7):1159-1179.

23. Stratford PW, Kennedy DM, Woodhouse LJ. Performance Measures Provide Assessments of Pain and Function in People With Advanced Osteoarthritis of the Hip or Knee. Phys Ther 2006; 86(11):1489-96.

24. Ateef M, Kulandaivelan S, Tahseen S. Test-retest Reliability and Correlates of 6-minute Walk Test in Patients. Indian J Rheumatol 2016; 11(4): 192-196.

25. Hall M, Hinman RS, van der Esch M, Is the relationship between increased knee muscle strength and improved physical function following exercise dependent on baseline physical function status? Arthritis Res Ther 2017;19(1):271.

26. Krauss I, Katzmarek U, Rieger MA, Sudeck G. Motives for physical exercise participation as a basis for the development of patient-oriented exercise interventions in osteoarthritis: a cross-sectional study. Eur J Phys Rehabil Med 2017; 53(4): 590-602.
27. Bakilan F, Armagan O, Ozgen M, Tascioglu F, Bolluk O, Alatas O. Effects of native type II collagen treatment on knee osteoarthritis: A Randomized Controlled Trial. Eurasian J Med 2016; 48(2):95-101.

28. Coriolano K, Aiken A, Harrison M, Pukall C, Brouwer B, Groll D. Changes in knee pain, perceived need for surgery, physical function and quality of life after dietary weight loss in obese women diagnosed with knee osteoarthritis. Osteoarthritis Cartilage. 2013; 21(4):S261-S312.

29. Castrogiovanni P, Trovato FM, Loreto C, Nsir H, Szychlinska MA, Musumeci G. Nutraceutical Supplements in the Management and Prevention of Osteoarthritis. Int J Mol Sci 2016:17(12): 2042.

30. Fusini F, Bisicchia S, Bottegoni C, Gigante A, Zanchini F, Busilacchi A. Nutraceutical supplement in the management of tendinopathies: a systematic review. Muscles Ligaments Tendons J 2016; 6(1):48-57. 